\title{
Systematic errors specific to a Snapshot Mueller Matrix
}

\section{Polarimeter}

\author{
Matthieu Dubreuil $^{1,2}$, Sylvain Rivet ${ }^{1,2}$, Bernard Le Jeune ${ }^{1,2}$ and Jack Cariou ${ }^{1,2}$. \\ 1) Université Européenne de Bretagne, France
}

2) Université de Brest, Laboratoire de Spectrométrie et Optique Laser (E.A. 938), 6 Avenue le Gorgeu, C.S. 93837, 29238 Brest Cedex, France

*Corresponding author: matthieu.dubreuil@univ-brest.fr

This paper deals with systematic errors specific to a snapshot Mueller matrix polarimeter by wavelength polarization coding. Their origins and effects are highlighted, and solutions for correction and stabilization are proposed. The different effects induced by them are evidenced by experimental results acquired with a given set-up and theoretical simulations carried out for more general cases. We distinguish the errors linked to some imperfection of elements in the experimental set-up from those linked to the sample under study.@ 2008 Optical Society of America.

OCIS codes: (230.5440) Polarization-selective devices, (260.5430) Polarization.

\section{1- Introduction}


An interesting way to optically characterize a material is to obtain its polarimetric signature via the measurement of its Mueller matrix. Since long full Mueller matrix polarimeters have been developed with various ways to generate and analyze polarization states. With all of these instruments, the control of imperfections in the polarizing elements at work in a given setup is necessary to achieve a consistent physical interpretation of the measured Mueller matrices [1]-[3], indeed, these defects are at the origin of systematic errors. The issue is the same with a Snapshot Mueller Matrix Polarimeter (SMMP).

We previously reported on the experimental feasibility of an SMMP by wavelength polarization coding [4]. The principle of the method is to generate several polarization states with a broadband spectrum source and birefringent retarders [5],[6]. On condition to use retarders with a well-chosen thickness ratio between them in order to generate a sufficient number of polarization states, one can use the signal issued from a spectrometer (a grating and a CCD camera) to extract the 16 coefficients of a Mueller matrix. The measurement time is thus only reduced to the acquisition time of the detection system. Let us consider the configuration described in Fig. 1 and denoted as $(e, e, 5 e, 5 e)$ in reference to the thickness of the retarders 1, 2, 3 and 4.

The signal, $I(\lambda)$, given by the spectrometer is periodic, and for a well suited analysis window, it can be expressed as:

$$
I(\lambda)=s(\lambda) \cdot \operatorname{Re}\left[g_{0}+\sum_{n=1}^{12}\left(g_{n}+i h_{n}\right) \cdot e^{i\left(n f_{0} \lambda\right)}\right]
$$

where $s(\lambda)$ is the shape of the spectrum, $g_{n}$ and $h_{n}$ are linear combinations of the Mueller coefficients $\left(m_{i j}\right)$, and $f_{0}$ is the fundamental frequency of the signal associated to the reference thickness termed here $e$. The signal is composed of 13 frequencies (from 0 to $12 f_{0}$ ). The extraction of the information is made in the Fourier domain, and thus $g_{n}$ and $h_{n}$ represent the 
magnitudes of the Fourier peaks in the real part and the imaginary part, respectively. Table 1 gives the relationships established between the magnitudes of the Fourier peaks and the Mueller coefficients in the case of the ideal configuration.

Because of the simplicity of the experimental set-up (polarizers, calcite wave-plates...), in this study the system was calibrated via a model of linear birefringent retarders whose retardance is proportional to the wavelength as described in [4]. This, of course, implies to be aware of the possible deviations from this model so as to identify and correct them. This paper is aimed at listing the causes of systematic errors liable to appear in the case of this new kind of Mueller matrix polarimeter. Awareness of the impact of such errors is strongly advised to further correct them in order to get sufficiently accurate results. Section 2 deals with the systematic errors associated to elements in the experimental set-up (retarders, polarizers, spectrometer), whereas Section 3 sets out the possible deviations generated by the sample under study.

\section{2- Systematic errors associated to the set-up}

\section{2-1-Thickness errors}

As thickness errors, $\Delta e$, on birefringent retarders are an important source of systematic error for an SMMP, it is worth discussing their consequences. The relationships established between the Fourier peaks (real and imaginary parts) and the Mueller coefficients were presented in Table 1 for the ideal configuration $(e, e, 5 e, 5 e)$. But, one should be aware of the existence of some uncertainty on retarder thickness (especially for calcite plates) in relation with their manufacturing process. Two consequences appear from thickness errors.

Firstly, as the position of the signal, $I(\lambda)$, in the analysis window depends on the reference thickness, $e$, any uncertainty on $e$ will affect the value of the ratio between the real and imaginary 
parts. Indeed, a sinusoidal signal can be interpreted as a sine, or cosine, function according to its position in the analysis window. As a consequence, an additional phase, $\phi_{w}$, has to be considered.

Secondly, the thicknesses of retarders 2,3 and 4 are not strictly integer multiple of the reference thickness $e$. So, let us denote as $\left(e, e+\Delta e_{2}, 5 e+\Delta e_{3}, 5 e+\Delta e_{4}\right)$ the true configuration and as $\phi_{2}, \phi_{3}, \phi_{4}$ the phases associated to the thickness errors, $\Delta e_{2}, \Delta e_{3}, \Delta e_{4}$ through the relationship: $\phi_{p}=\frac{2 \pi \Delta n \Delta e_{p}}{\lambda_{0}}(p=2,3,4)$ where $\Delta n$ is the birefringence of the plates, and $\lambda_{0}$ is the central wavelength of the analysis window.

Let us consider an error on each retarder of the set-up; the intensity on the spectrometer is then:

$$
I(\lambda)=s(\lambda) \cdot \operatorname{Re}\left[g_{0}^{\prime}+\sum_{n=1}^{12} e^{i \varphi_{n}} \cdot\left(g^{\prime}{ }_{n}+i h^{\prime}{ }_{n}\right) \cdot e^{i . n\left(f_{0} \lambda+\phi_{w}\right)}\right]
$$

where $\varphi_{n}$ is an additional phase, which depends on $\phi_{p}(p=2,3,4), g^{\prime}{ }_{n}$ and $h_{n}{ }_{n}$ are functions that depend on the Mueller coefficients, $m_{i j}$, and phases, $\phi_{p}$. Thus, Eq.(2) allows one to establish new relationships between the Fourier peaks and the Mueller coefficients. They are given in Table 2.

Table 3 shows how the Mueller coefficients are affected by the values of the phases, $\phi_{w}$ and $\phi_{p}(p=2,3,4)$, in the case of two different media.

It is clear that Mueller coefficients, especially those that involve high frequency peaks ( $10 f_{0}$ to $12 f_{0}$ ), e.g. $m_{03}, m_{30}, m_{02}, m_{20}, m_{23}$ and $m_{32}$, are strongly dependent on the value of $\phi_{w}$. In the case of the true configuration, thickness errors lead to $\phi_{w}$ and $\phi_{p}$ values between $-\pi$ and $\pi$ and could be much higher than $0.01 \mathrm{rad}$. It is, thus, paramount to calculate and include them into the model. Table 3 also gives an insight into the accuracy required on the extraction of $\phi_{w}$ and $\phi_{p}$ : for example, when the accuracy on $\phi_{p}$ values is less than $0.01 \mathrm{rad}$, the maximum absolute error on 
Mueller coefficients is less than 0.01 . On the other hand, for the same absolute error, the accuracy on the value of $\phi_{w}$ must be less than $0.001 \mathrm{rad}$.

From the output data $I(\lambda)$ of a known-sample, the relationships given in Table 2 can be used to retrieve the values of all of the phases. Indeed, several equations can be generated by measuring the argument of each peak in the Fourier domain. In our opinion, to retrieve all of the phases (calibration), it is worth proceeding through two steps as follows: at first, the intensity of the signal produced by vacuum (a well-known sample) is used:

$$
\begin{aligned}
16 I^{\text {vacuum }}(\lambda)= & 3+\cos \left[2\left(\phi_{w}+f_{0} \lambda\right)+\phi_{2}+\phi_{3}-\phi_{4}\right]-2 \cos \left[4\left(\phi_{w}+f_{0} \lambda\right)+\phi_{4}\right] \\
& -2 \cos \left[6\left(\phi_{w}+f_{0} \lambda\right)+\phi_{4}\right]+\cos \left[10\left(\phi_{w}+f_{0} \lambda\right)+\phi_{2}+\phi_{3}+\phi_{4}\right] \\
& -\cos \left[12\left(\phi_{w}+f_{0} \lambda\right)+\phi_{2}+\phi_{3}+\phi_{4}\right]
\end{aligned}
$$

Indeed, in the Fourier domain, the generation of six peaks from this signal allows one to calculate $\phi_{w}, \phi_{2+} \phi_{3}$ and $\phi_{4}$. Then, as the phases $\phi_{2}$ and $\phi_{3}$ have to be distinguished, another sample is needed. It can be, for example, a linear polarizer oriented at an azimuth different from $0^{\circ}$ to generate more equations. The linear polarizer used here was oriented at $30^{\circ}$; its signal in the Fourier domain was thus composed of 13 peaks.

Table 4 gives the experimental Mueller matrices obtained under the previous conditions for vacuum and a linear partial polarizer oriented at $30^{\circ}$, before and after corrections by the phases, $\phi_{w}$ and $\phi_{p}$.

Extraction of the phases from the signals produced by these media led to $\phi_{w}=-0.1251 \mathrm{rad}, \phi_{2}=-0.0498 \mathrm{rad}, \phi_{3}=-1.7236 \mathrm{rad}, \phi_{4}=-2.3817 \mathrm{rad}$. One should note that the highest absolute error on the Mueller coefficients is less than 0.02 . In the case of much higher thickness errors, the calibration would be ineffective because of the possible generation of non $f_{0}$-multiple frequencies. Nevertheless, the different runs of simulations that we carried out taught 
us that a thickness error of less than $1 \%$ from the ideal configuration permits the application of the previous calibration method to extract all the Mueller coefficients with a maximum absolute error below 0.02 (efficient calibration).

In conclusion, the correction of the phases associated to thickness errors toward the theoretical configuration is a prerequisite. But, one should be aware that such a correction is only valid at a given time because of possible evolution in environmental conditions.

\section{2-2-Sensitivity to environmental changes}

Calcite retarder plates are sensitive to environmental changes, especially fluctuations in temperature. Their thickness is, thus, strongly affected by thermal expansion, and thus calibrated values will change. As the thickness ratio between calcite plates is known, it would be worth making a simple measurement of temperature to recalculate the phases. This consideration drove us to investigate the relevance of using a model-dependent correction (linear thermal expansion). To gain more insight into the linearity of calcite plate length-expansion, one of the calcite retarders, of thickness, $5 e$, was set between two crossed-polarizers. The signal detected on the spectrometer being periodic at the frequency, $5 f_{0}$, it generates a single peak in the Fourier domain. Since its argument is dependent upon the retarder thickness, plotting the evolution of the argument value versus temperature (Fig.2) gives insight into the thickness evolution with temperature. Figure 2 shows that the evolutions of thickness and temperature are nearly alike, but it also makes appear an effect of hysteresis: for example, at $\mathrm{T}=22^{\circ} \mathrm{C}$ over the first rise of temperature, the phase value is about $0.10 \mathrm{rad}$ against about $0.37 \mathrm{rad}$ over the second rise. This difference may result from a mechanical strain by the mount of the calcite retarder. Thus, for the plates, the lack of total freedom for thermal expansion forbids the use of the linear model under test. This led us to carry out a no-sample experiment in order to gain insight into the temperature 
dependency of the values of $\phi_{w}, \phi_{2+} \phi_{3}$ and $\phi_{4}$, (Fig. 3). As shown above, a prerequisite to an efficient calibration (maximum absolute error on the measured Mueller coefficients less than 0.02) is phase correction. For $\phi_{p}$, a variation below 0.01 leads to an absolute error of 0.01 on $m_{i j}$. This is the case when the temperature varies of about $0.2^{\circ} \mathrm{C}$. Nevertheless, with such a difference, the evolution of $\phi_{w}$ (around 0.02) is sufficient to strongly affect the Mueller matrix (Table 3). So, it is paramount to retrieve the value of $\phi_{w}$ at any time $t$. Whatever the medium under study, this method allows a direct measurement of $\phi_{w}$ through, for example, the use of the arguments of $4 f_{0}$ and $6 f_{0}$ peaks (Eq.(2) and Table 2). One should note that this implies that $m_{11}$ is different from zero. Figure 4 illustrates how the Mueller matrix coefficients are affected by a slow temperature change of $0.2^{\circ} \mathrm{C}$ and highlights the stabilization of all of the Mueller coefficients induced by the $\phi_{w}$ correction. It also shows that, among them and as expected, $m_{03}$, $m_{23}$ and $m_{32}$ are the most temperature-dependent: indeed, their variations with temperature are slow, but important. On the other hand, the quick variations of $m_{i j}$ are likely associated to random noise.

One of the aim of the SMMP is to allow one to follow the time-evolution of Mueller matrices, $M(t)$. Two solutions result from the above study: i) the user carries out a calibration as described in $\S 2.1$ just before following the time-evolution of an unknown medium; under these conditions and on condition that the range of temperature fluctuations is less than $0.2^{\circ} \mathrm{C}$ throughout the experiment, the measurement of $\phi_{w}$ at any time, $t$, constitutes an efficient calibration. ii) The set-up temperature is kept at the same value $\pm 0.2^{\circ} \mathrm{C}$. The calibrated values of $\phi_{p}$ are thus stable enough (several days), and a simple measurement of $\phi_{w}$ at any time, $t$, during $M(t)$ follow-up guarantees that the maximum absolute error on the measured Mueller coefficients is less than 0.02 . 


\section{2-3-Misalignment errors}

The SMMP configuration under study assumes the alignment of optical elements as described in Fig.1. But, as they cannot be aligned in this ideal configuration, it is worth studying the overall impact by the misalignment errors on the four retarders, 1, 2, 3 and 4, and the output polarizer $\left(\Delta \theta_{1}, \Delta \theta_{2}, \Delta \theta_{3}, \Delta \theta_{4}\right.$ and $\Delta \theta_{p o l}$, respectively). Because of the high number of optical elements at play, the development of the theoretical expressions relative to the misalignmentinduced errors would be too complicated to provide insight. A thorough analysis of numerical simulations is a way to investigate the impact by these errors. Table 5 presents the results of simulations run with vacuum as medium and a misalignment error of $0.5^{\circ}$, or $0.1^{\circ}$, on each element. They highlight that a combination of errors may lead to an important error on the Mueller coefficients. For example, Table 5 shows that $m_{12}$, which was expected to be null, is equal to -0.05 . To avoid this problem, a solution could be the inclusion of angular errors into the model, but these errors are very difficult to extract in the case of an SMMP. On condition the misalignment of the optical elements is less than $0.1^{\circ}$ thanks to the use, for example, of precise angular controllers, the accuracy on the Mueller coefficients should be less than 0.01 .

\section{2-4- Effect by the spectrometer}

For detection purpose, an SMMP device is equipped with a diffraction grating coupled to a CCD camera in charge of recording the spectrum, $I(\lambda)$. But, two sources of systematic errors are associated to this detection system. Firstly, the response of the spectrometer (due to the numerical aperture of the optics) leads to an attenuation of Fourier peaks as previously discussed in [4]; a no-sample acquisition before the measurement of an unknown medium allows one to get a stable enough response, even in the case of fluctuations in the set-up environment. Secondly the grating acts as a partial polarizer, but this has no effect on the signal, except when the output 
polarizer is also a partial polarizer. In that case, systematic errors may occur and affect the accuracy of the Mueller matrix. This problem can be avoided by: i) either depolarizing the light before its interaction with the detector through the use of a depolarizing fiber or that of a scattering medium set in front of the spectrometer, ii) or aligning properly the axis of the output polarizer on the grating one.

\section{2-5- Random noise}

With the method reported in [4], the time required for the acquisition of the full Mueller matrix of a given sample should be very short $(<\mu s)$. This means that the random noise will be interpreted as a systematic error. One should note that, with the usual methods, the random noise is reduced through a time-consuming accumulation of data incompatible with SMMP. Therefore, the inevitable random noise has to be minimized.

Three parameters play a role in the signal-to-noise ratio: the thickness-configuration for the retarders, the global response of the detection system and the acquisition time of the detector. The choice of the SMMP thickness-configuration that propagates the minimum random noise was discussed in [7]. Nevertheless, this choice is independent of the detection system sampling. It is worth recalling that the amplitude of Fourier peaks is sampling rate-dependent, which means that any enhancement of the resolution by the spectrometer will likely be accompanied with an increase of the signal-to-noise ratio. Furthermore, the fundamental frequency $f_{0}$ is dependent on the reference thickness $e$, and thus the value chosen for $e$ must be such that the highest generated frequency $\left(12 f_{0}\right.$ in the case of the configuration $\left.(e, e, 5 e, 5 e)\right)$ is not too much affected by the response of the spectrometer. Lastly, the higher the intensity of the luminous flux is, the shorter the acquisition time is, and thus a very short acquisition time requires the use of a coherent source. The signal-to-noise ratio due to the acquisition time is dependent on the nature 
(transparent or diffusing) of the medium under study. The acquisition time has to be adapted when necessary.

\section{3- Sample-associated limitations}

One should be aware that, with SMMP, a non-uniform spectral response of the sample under study within the analysis window may induce an error on the measurement of the Mueller matrix. This limitation creates an effect similar to a systematic error on the experimental set-up.

\section{3-1-Evolution with the wavelength}

In the SMMP model, the Mueller coefficients are assumed to be wavelength-independent. This study is, indeed, aimed at demonstrating the feasibility of a monochromatic snapshot measurement of the Mueller matrix of a given sample. If $m_{i j}$ variations with the wavelength are important in the analysis window, Fourier peaks are widened, and thus overlapped, which generates deviations on the Mueller matrix. This consideration drove us to choose a detection system with a narrow analysis window (10-nm wide). Nevertheless, it is not sufficient as shown the following study.

In the case, for example, of a retarder, what is the relative optical path-length difference, $\Delta n e$, that permits a retrieval of $m_{i j}$ values with the maximum absolute error below $1 \%$ on its extracted physical parameters? Table 6 presents the results of simulations run with a quarterwave plate at the central wavelength, $\lambda_{c}$, and at different orders $\left((\Delta n e) \lambda_{c / 4}=\lambda_{c} / 4+k \lambda_{c}\right)$. Let us denote as $\Delta \phi_{\lambda c / 4}$ the variation of the quarter-wave plate retardance on the analysis window and as $\Delta \phi_{\text {coding }}$ that of the coding system retarder of thickness $e ; \Delta \phi_{\lambda c / 4}$ is increasing with the order of the plate, $k$. The ratio between $\Delta \phi_{i c / 4}$ and $\Delta \phi_{\text {coding }}$ represents the ratio between the relative optical path-length difference of the quarter wave plate $(\Delta n e)_{\lambda c / 4}$ and that of the coding retarders 
$(\Delta n e)_{\text {coding. }}$ Table 6 shows a deterioration of the parameters calculated from the polar decomposition of the Mueller matrix [8] when $\Delta \phi_{\lambda c / 4}$ is no longer negligible toward $\Delta \phi_{\text {coding. }}$. In fact, a $(\Delta n e)_{\lambda c / 4}$-to- $(\Delta n e)_{\text {coding }}$ ratio around $2 \%$ leads to an error of $1 \%$ on $P_{D}$ (depolarization index) and $\alpha$ (azimuthal angle) and below $1 \%$ on $R$ (retardance). For instance, for the SMMP in the proposed configuration, the plates were made of calcite $\left(\Delta n_{\text {coding }}=0.166\right)$, and their thickness was $e_{\text {coding }} \approx 2.08 \mathrm{~mm}$. Consequently, for retarders with a $\Delta n e$ value less than $7 \mu \mathrm{m}$, the error on their measurement will be less than $1 \%$. It is thus interesting to use thick retarders for the coding system in order to extend the possibility of using the SMMP with a good accuracy, even for medium whose $m_{i j}$ coefficients vary with the wavelength.

\section{3-2-Multiple wave interferences}

A spatially coherent source associated to a multi-spectral detection can be at the origin of interferences on the detector. Indeed, because of the possible multiple reflections in the sample under study, interferences such as those observed with a Fabry-Perot interferometer can be produced. They lead to another kind of bias when the periodicity of the signal given by the interference phenomenon generates a frequency on the spectral analysis window ( 0 to $12 f_{0}$ in the Fourier domain).

Let us consider a quartz wave plate cut perpendicular to the optical axis (circular birefringent with a rotatory power of $\theta \approx-12^{\circ}$ at $830 \mathrm{~nm}$ for a $\approx 1 \mathrm{~mm}$-length plate). No antireflection coating had been applied to this plate. Consequently, multiple reflections on the two faces may generate interferences. Figure 5 shows the signal, $T_{F P}(\lambda)$, produced by this plate when it is set between two crossed polarizers.

The periodic signal produced by interferences acts like a systematic error in the reconstruction. The effect can be interpreted as a transmission function, $T_{F P}(\lambda)$, that overlays the 
modulated signal, $I(\lambda)$. When the signal, $I(\lambda)$, is divided by $T_{F P}(\lambda)$, accurate results are obtained. Table 7 presents the experimental Mueller matrix of the quartz wave plate with and without correction.

The effect of the correction is obvious on the Mueller coefficient, $m_{31}$, which was expected to be null. To gain insight into the global efficiency of the correction on the Mueller matrix, let us use the Frobenius norm [9], $\|\Delta M\|_{F}=\sqrt{\sum_{i j}\left|m_{i j}^{e x p}-m_{i j}^{t h}\right|^{2}}$, to compare the experimental Mueller matrix against the theoretical one. Table 7 highlights the significant improvement produced by the correction.

\section{4- Conclusion}

This paper listed the possible systematic errors associated to a Snapshot Mueller Matrix Polarimeter (SMMP) and showed how to compensate for them. As the errors associated to the set-up are mostly dependent on the accuracy about retarder thickness, we developed, here, a method to calibrate the system and stabilize it toward thermal evolution. The presence of other kinds of systematic errors (misalignment errors, influence of the spectrometer) drove us to propose methods to limit their consequences. Moreover, the impact of random noise, considered here as a systematic error because of snapshot acquisition, has to be limited. Besides, one should be aware that the errors liable to be generated by the non-uniform spectral response of the sample in the analysis window constitute a limitation to the application field of an SMMP.

The assets of a SMMP are the possibility of short-time acquisitions and the compactness of the experimental set-up. The simplicity of elements naturally involves the use of a model and the study of the possible deviations. However, in the future, it could be worth considering the 
set-up as a totally unknown system and calculating its response to well-known samples in order to focus on accuracy enhancement and mastery of the impact by temperature fluctuations.

\section{Acknowledgments}

The authors thank M. G.Zion for the technical assistance and Dr M.P.Friocourt for help in the preparation of the English manuscript.

\section{References}

1. B. Boulbry, B. Le Jeune, F.Pellen, J.Cariou and J. Lotrian, "Error analysis and calibration of a spectroscopic Mueller matrix polarimeter using a short-pulse laser source", Meas.Sci.Technol. 13, 1563-1573 (2002)

2. D.H. Goldstein and R.A. Chipman, "Error analysis of a Mueller matrix polarimeter," J. Opt. Soc. Am. A 7, 693-700 (1990)

3. L. Broch, A. En Naciri, and L. Johann, "Systematic errors for a Mueller matrix dual rotating compensator ellipsometer", Opt. Express 16, 8814-8824 (2008)

4. M.Dubreuil, S.Rivet, B.Le Jeune and J.Cariou, "Snapshot Mueller matrix polarimeter by wavelength polarization coding," Opt. Express 15, 13660-13668 (2007)

5. K. Oka and T. Kato, "Spectroscopic polarimetry with a channeled spectrum," Opt. Lett. 24, $1475-1477$ (1999)

6. N. Hagen, K. Oka, and E. L. Dereniak, "Snapshot Mueller matrix spectropolarimeter," Opt. Lett. 32, 2100-2102 (2007)

7. P. Lemaillet, S. Rivet, and B. Le Jeune, "Optimization of a snapshot Mueller matrix polarimeter," Opt. Lett. 33, 144-146 (2008) 
8. J.J.Gil, E.Bernabeu, "Obtainment of the polarizing and retardation parameters of a nondepolarizing optical system from polar decomposition of its Mueller matrix", Optik(Stuttgart) 76, 67-71 (1987)

9. D.G.M.Anderson and R.Barakat, "Necessary and sufficient conditions for a Mueller matrix to be derivable from a Jones matrix," J. Opt. Soc. Am. A 11, 2305-2319 (1994)

\section{List of Figure Captions}

Fig.1: Experimental set-up for an SMMP in the configuration $(e, e, 5 e, 5 e)$ with $e$ as the reference thickness of the first retarder.

Fig.2: Evolution of the argument $\left(\phi_{w}\right)$ of the peak at $5 f_{0}$ given by a signal of one calcite retarder of thickness $5 e$ set between crossed-polarizers versus temperature.

Fig.3: Evolution of the phases, $\phi_{w}, \phi_{2}+\phi_{3}$ and $\phi_{4}$, calculated with a no-sample measurement versus temperature. Phases were adapted so as to be null at $\mathrm{t}=0$.

Fig.4: Evolution of the Mueller coefficients of a no-sample measurement (vacuum) versus temperature over the first 15 minutes of experiment of Fig.3. Solid curves represent Mueller coefficients with no correction, and dashed curves show them after correction by $\phi_{w}$.

Fig.5: Intensity spectrum $T_{F P}(\lambda)$ of the quartz wave plate (circular birefringent) between crossedpolarizers 


\section{List of Table captions}

Table 1: Relationships between the magnitudes of the peaks - real part $\left(g_{n}\right)$ and imaginary part $\left(h_{n}\right)$ - and the Mueller coefficients $\left(m_{i j}\right)$ in the Fourier domain. It is given for the ideal configuration $(e, e, 5 e, 5 e)$.

Table 2: Relationships between the magnitudes of the peaks - real part $\left(g^{\prime}{ }_{n}\right)$ and imaginary part $\left(h_{n}^{\prime}\right)$ - and the Mueller coefficients $\left(m_{i j}\right)$ in the Fourier domain. It is given for the true configuration $\left(e, e+\Delta e_{2}, 5 e+\Delta e_{3}, 5 e+\Delta e_{4}\right)$.

Table 3: Influence of the phases, $\phi_{w}, \phi_{2}, \phi_{3}$ and $\phi_{4}$, on the Mueller matrix for vacuum and a linear polarizer at $30^{\circ}$. Phases are expressed in radians. In the ideal case (no phase errors) and for the linear polarizer at $30^{\circ}$, the value of the polarization parameters depolarization index $\left(P_{D}\right)$, diattenuation $(D)$ and retardance $(R)$ are $\left(P_{D}=1, D=0, R=0\right)$. When $\left(\phi_{p}=0, \phi_{w}=0.01\right)$, these values become $\left(P_{D}=0.989, D=0.999, R=0.58\right)$.

Table 4: Experimental Mueller matrix given by the SMMP for vacuum and a linear partial polarizer at $30^{\circ}$ : theoretical, without corrections by $\phi_{w}, \phi_{2}, \phi_{3}$ and $\phi_{4}$ and with corrections by $\phi_{w}$, $\phi_{2}, \phi_{3}$ and $\phi_{4}$. All matrices are normalized by $m_{00}$. The experimental set-up is composed of two calcite plates $(\Delta n=0.166)$ for the coding system $(e=2.08 \mathrm{~mm} \pm 0.01 \mathrm{~mm})$ and two calcite plates for the decoding system $(e=10.4 \mathrm{~mm} \pm 0.01 \mathrm{~mm})$. The source is a broadband spectrum source with $\lambda_{0}=829 \mathrm{~nm}$, and the analysis window of the detection system is $\Delta \lambda=10 \mathrm{~nm}$ sampled with 512 pixels. 
Table 5: Simulation of the influence of the misalignment errors, $\Delta \theta_{1}, \Delta \theta_{2}, \Delta \theta_{3}, \Delta \theta_{4}$ and $\Delta \theta_{p o l}$, on the Mueller matrix for vacuum.

Table 6: Simulation of a quarter wave plate $\left(R=90^{\circ}, \alpha=20^{\circ}\right)$ at different orders. Depolarization index $P_{D}$, retardance $R$ and azimuthal angle $\alpha$ are calculated. The ratio between the evolution with the wavelength of the quarter wave plate retardance and the evolution with the wavelength of the reference coding plate retardance is given.

Table 7: Experimental Mueller matrix for the quartz wave plate: theoretical, with no correction, and with correction. 


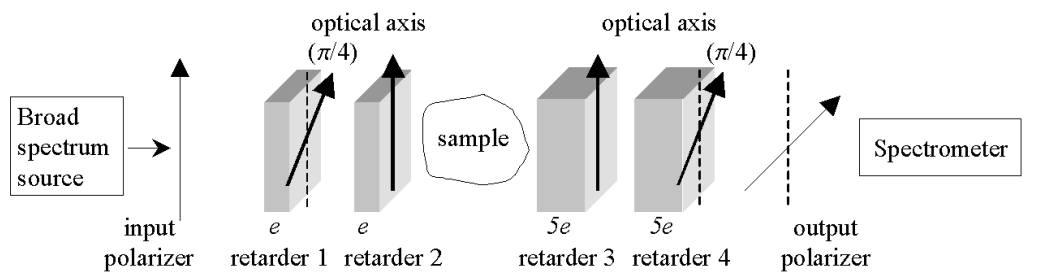

Fig.1: Experimental set-up for an SMMP in the configuration $(e, e, 5 e, 5 e)$ with $e$ as the reference thickness of the first retarder. 


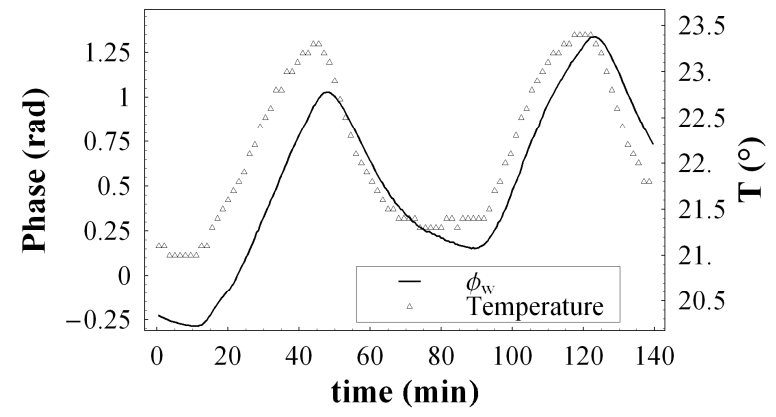

Fig.2: Evolution of the argument $\left(\phi_{w}\right)$ of the peak at $5 f_{0}$ given by a signal of one calcite retarder of thickness $5 e$ set between crossed-polarizers versus temperature. 


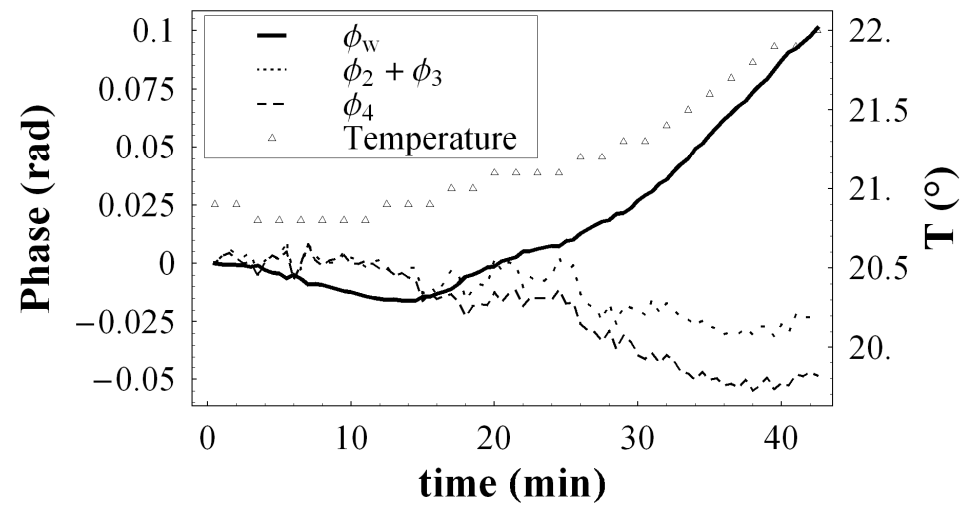

Fig.3: Evolution of the phases, $\phi_{w}, \phi_{2}+\phi_{3}$ and $\phi_{4}$, calculated with a no-sample measurement versus temperature. Phases were adapted so as to be null at $\mathrm{t}=0$. 

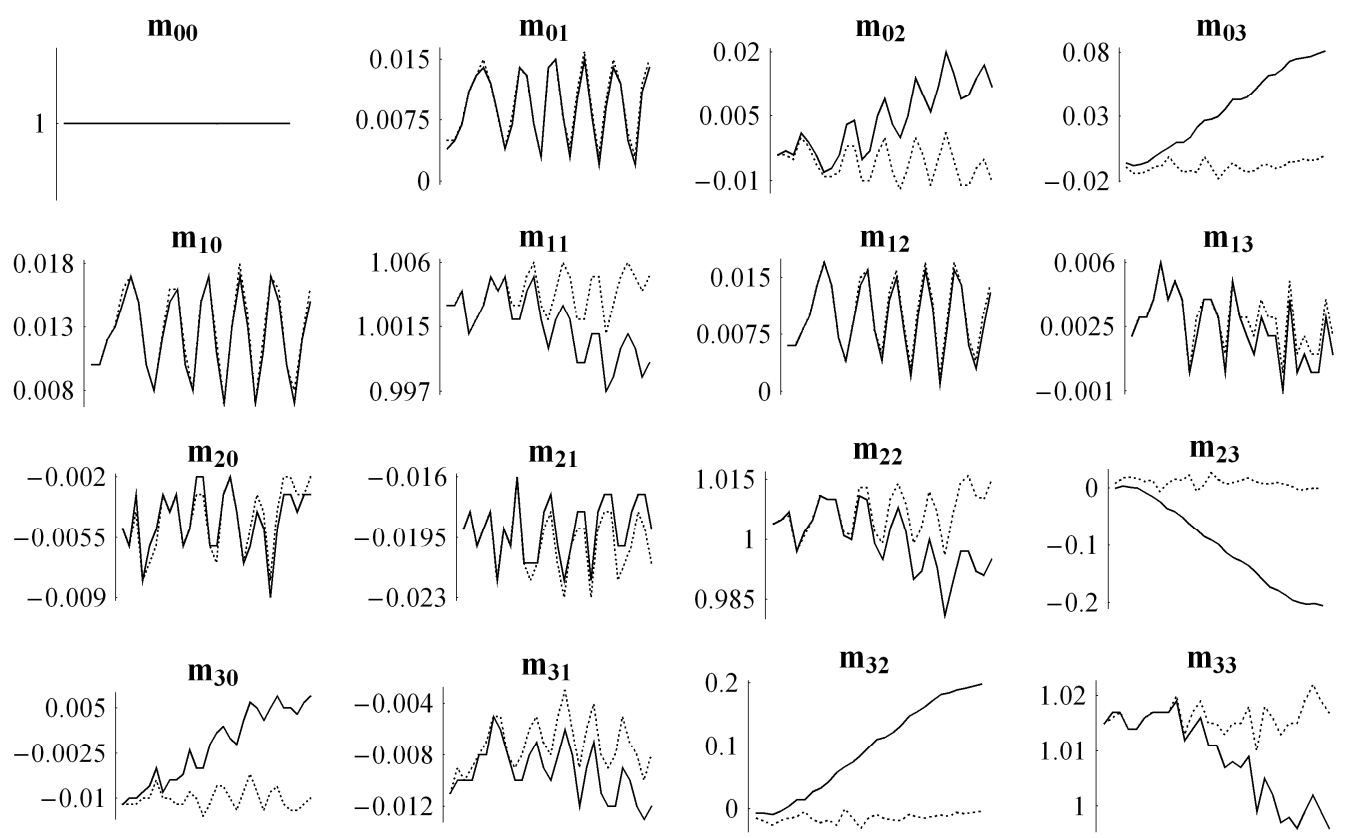

Fig.4: Evolution of the Mueller coefficients of a no-sample measurement (vacuum) versus temperature over the first 15 minutes of experiment of Fig.3. Solid curves represent Mueller coefficients with no correction, and dashed curves show them after correction by $\phi_{w}$. 


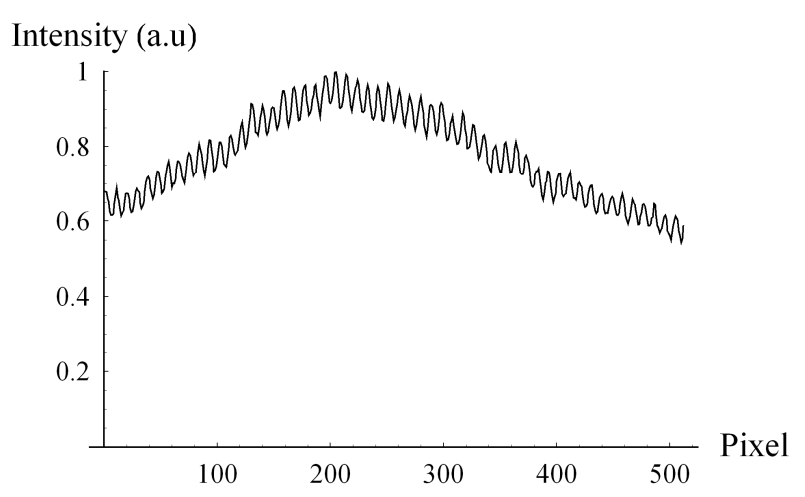

Fig.5: Intensity spectrum $T_{F P}(\lambda)$ of the quartz wave plate (circular birefringent) between crossedpolarizers 
Table 1: Relationships between the magnitudes of the peaks - real part $\left(g_{n}\right)$ and imaginary part $\left(h_{n}\right)$ - and the Mueller coefficients $\left(m_{i j}\right)$ in the Fourier domain. It is given for the ideal configuration $(e, e, 5 e, 5 e)$.

\begin{tabular}{cccc}
\hline frequency & $64 \mathrm{~g}_{\mathrm{n}}$ & & $64 \mathrm{~h}_{\mathrm{n}}$ \\
\cline { 1 - 2 } $\mathrm{f}_{0}$ & $16 m_{00}+8 m_{02}-8 m_{20}-4 m_{22}$ & 0 \\
$2 \mathrm{f}_{0}$ & $8 m_{01}-4 m_{21}$ & 0 \\
$3 \mathrm{f}_{0}$ & $-4 m_{02}+2 m_{22}$ & $-4 m_{03}+2 m_{23}$ \\
$4 \mathrm{f}_{0}$ & $2 m_{12}$ & $-2 m_{13}$ \\
$5 \mathrm{f}_{0}$ & $-4 m_{11}$ & 0 \\
$6 \mathrm{f}_{0}$ & $-8 m_{10}-4 m_{12}$ & 0 \\
$7 \mathrm{f}_{0}$ & $-4 m_{11}$ & 0 \\
$8 \mathrm{f}_{0}$ & $2 m_{12}$ & $2 m_{13}$ \\
$9 \mathrm{f}_{0}$ & $-m_{22}+m_{33}$ & $m_{23}+m_{32}$ \\
$10 \mathrm{f}_{0}$ & $2 m_{21}$ & $-2 m_{31}$ \\
$11 \mathrm{f}_{0}$ & $4 m_{20}+2 m_{22}$ & $-4 m_{30}-2 m_{32}$ \\
$12 \mathrm{f}_{0}$ & $2 m_{21}$ & $-2 m_{31}$ \\
\hline
\end{tabular}


Table 2: Relationships between the magnitudes of the peaks - real part $\left(g^{\prime}{ }_{n}\right)$ and imaginary part $\left(h_{n}^{\prime}\right)$ - and the Mueller coefficients $\left(m_{i j}\right)$ in the Fourier domain. It is given for the true configuration $\left(e, e+\Delta e_{2}, 5 e+\Delta e_{3}, 5 e+\Delta e_{4}\right)$.

\begin{tabular}{|c|c|c|c|}
\hline frequency & $\varphi_{\mathrm{n}}$ & $64 \mathrm{~g}_{\mathrm{n}}$ & $64 h_{n}^{\prime}$ \\
\hline 0 & 0 & $\begin{array}{l}16 m_{00}+8 m_{02} \cos \left(\phi_{2}\right)+8 m_{03} \sin \left(\phi_{2}\right) \\
\quad-8 m_{20} \cos \left(\phi_{3}-\phi_{4}\right)+8 m_{30} \sin \left(\phi_{3}-\phi_{4}\right) \\
\quad-4 m_{22} \cos \left(\phi_{2}\right) \cos \left(\phi_{3}-\phi_{4}\right) \\
\quad+4 m_{33} \sin \left(\phi_{2}\right) \sin \left(\phi_{3}-\phi_{4}\right) \\
\quad-4 m_{23} \sin \left(\phi_{2}\right) \cos \left(\phi_{3}-\phi_{4}\right) \\
\quad+4 m_{32} \cos \left(\phi_{2}\right) \sin \left(\phi_{3}-\phi_{4}\right)\end{array}$ & 0 \\
\hline $\mathrm{f}_{0}$ & 0 & $8 m_{01}-4 m_{21} \cos \left(\phi_{3}-\phi_{4}\right)+4 m_{31} \sin \left(\phi_{3}-\phi_{4}\right)$ & 0 \\
\hline $2 \mathrm{f}_{0}$ & $\phi_{2}$ & $\begin{array}{r}-4 m_{02}+2 m_{22} \cos \left(\phi_{3}-\phi_{4}\right) \\
-2 m_{23} \sin \left(\phi_{3}-\phi_{4}\right)\end{array}$ & $\begin{array}{r}-4 m_{03}-2 m_{33} \sin \left(\phi_{3}-\phi_{4}\right) \\
+2 m_{23} \cos \left(\phi_{3}-\phi_{4}\right)\end{array}$ \\
\hline $3 f_{0}$ & $-\phi_{2}+\phi_{4}$ & $2 m_{12}$ & $-2 m_{13}$ \\
\hline $4 f_{0}$ & $\phi_{4}$ & $-4 m_{11}$ & 0 \\
\hline $5 f_{0}$ & $\phi_{4}$ & $-8 m_{10}-4 m_{12} \cos \left(\phi_{2}\right)-4 m_{13} \sin \left(\phi_{2}\right)$ & 0 \\
\hline $6 f_{0}$ & $\phi_{4}$ & $-4 m_{11}$ & 0 \\
\hline $7 \mathrm{f}_{0}$ & $\phi_{2}+\phi_{4}$ & $2 m_{12}$ & $2 m_{13}$ \\
\hline $8 \mathrm{f}_{0}$ & $-\phi_{2}+\phi_{3}+\phi_{4}$ & $-m_{22}+m_{33}$ & $m_{23}+m_{32}$ \\
\hline $9 \mathrm{f}_{0}$ & $\phi_{3}+\phi_{4}$ & $2 m_{21}$ & $-2 m_{31}$ \\
\hline $10 \mathrm{f}_{0}$ & $\phi_{3}+\phi_{4}$ & $4 m_{20}+2 m_{22} \cos \left(\phi_{2}\right)+2 m_{23} \sin \left(\phi_{2}\right)$ & $-4 m_{30}-2 m_{33} \sin \left(\phi_{2}\right)-2 m_{32} \cos \left(\phi_{2}\right)$ \\
\hline $11 f_{0}$ & $\phi_{3}+\phi_{4}$ & $2 m_{21}$ & $-2 m_{31}$ \\
\hline $12 \mathrm{f}_{0}$ & $\phi_{2}+\phi_{3}+\phi_{4}$ & $-m_{22}-m_{33}$ & $-m_{23}+m_{32}$ \\
\hline
\end{tabular}


Table 3: Influence of the phases, $\phi_{w}, \phi_{2}, \phi_{3}$ and $\phi_{4}$, on the Mueller matrix for vacuum and a linear polarizer at $30^{\circ}$. Phases are expressed in radians. In the ideal case (no phase errors) and for the linear polarizer at $30^{\circ}$, the value of the polarization parameters depolarization index $\left(P_{D}\right)$, diattenuation $(D)$ and retardance $(R)$ are $\left(P_{D}=1, D=0, R=0\right)$. When $\left(\phi_{p}=0, \phi_{w}=0.01\right)$, these values become $\left(P_{D}=0.989, D=0.999, R=0.58\right)$.

$\phi_{2} \quad \begin{array}{lllcl}\phi_{3} & \phi_{4} & \phi_{\mathrm{w}} & \begin{array}{c}\text { Mueller Matrix for } \\ \text { vacuum }\end{array} & \begin{array}{c}\text { Mueller Matrix } \\ \text { for a linear polarizer } \\ \left(\boldsymbol{\theta}=\mathbf{3 0}^{\circ}\right)\end{array}\end{array}$

\begin{tabular}{|c|c|c|c|c|c|c|c|c|c|c|c|}
\hline 0 & 0 & 0 & 0 & & $\left(\begin{array}{ll}1 & 0 \\
0 & 1 \\
0 & 0 \\
0 & 0\end{array}\right.$ & $\left.\begin{array}{ll}0 & 0 \\
0 & 0 \\
1 & 0 \\
0 & 1\end{array}\right)$ & & $\left(\begin{array}{c}1 \\
-0.5 \\
0.866 \\
0\end{array}\right.$ & $\begin{array}{cc} & -0.5 \\
& 0.25 \\
6 & -0.433 \\
& 0\end{array}$ & $\begin{array}{cc} & 0.866 \\
-0.433 \\
3 & 0.75 \\
& 0\end{array}$ & $\left.\begin{array}{ll}3 & 0 \\
3 & 0 \\
& 0 \\
& 0\end{array}\right)$ \\
\hline 0.01 & 0 & 0 & 0 & $\left(\begin{array}{c}1 \\
0 \\
0.002 \\
0.004\end{array}\right.$ & $\begin{array}{c}0 \\
1.002 \\
0 \\
0\end{array}$ & $\begin{array}{c}0 \\
0 \\
1.001 \\
-0.01\end{array}$ & $\left.\begin{array}{c}0 \\
0 \\
1 \\
0.01 \\
1 \\
1.001\end{array}\right)$ & $\left(\begin{array}{cc}1 & - \\
-0.5 \\
0.866 \\
0\end{array}-\right.$ & $\begin{array}{c}-0.499 \\
0.249 \\
-0.432 \\
0\end{array}$ & $\begin{array}{c}0.864 \\
-0.432 \\
0.748 \\
0\end{array}$ & $\left.\begin{array}{c}0.009 \\
-0.004 \\
0.007 \\
0\end{array}\right)$ \\
\hline 0 & 0.01 & 0 & 0 & $\left(\begin{array}{l}1 \\
0 \\
0 \\
0\end{array}\right.$ & $\begin{array}{rr}0 & -0 . \\
1 & \\
0 & \\
0 & -0\end{array}$ & $\begin{array}{ll}03 & -0 \\
01 & \\
0 & \end{array}$ & $\left.\begin{array}{c}0.004 \\
0 \\
0.01 \\
1\end{array}\right)$ & $\left(\begin{array}{c}1 \\
-0.5 \\
0.866 \\
-0.009\end{array}\right.$ & $\begin{array}{cc} & -0.498 \\
& 0.25 \\
6 & -0.433 \\
9 & 0.004\end{array}$ & $\begin{array}{cc}8 & 0.863 \\
& -0.433 \\
33 & 0.75 \\
4 & -0.008\end{array}$ & $\left.\begin{array}{ll}3 & 0 \\
3 & 0 \\
& 0 \\
8 & 0\end{array}\right)$ \\
\hline 0 & 0 & 0.01 & 0 & $\left(\begin{array}{l}1 \\
0 \\
0 \\
0\end{array}\right.$ & $\begin{array}{lr}0 & -0 . \\
1 & \\
0 & \\
0 & -0\end{array}$ & $\begin{array}{ll}003 & -0 \\
01 & 0\end{array}$ & $\left.\begin{array}{c}0.004 \\
0 \\
0.01 \\
1\end{array}\right)$ & $\left(\begin{array}{c}1 \\
-0.5 \\
0.866 \\
-0.009\end{array}\right.$ & $\begin{array}{cc} & -0.498 \\
6 & 0.25 \\
6 & -0.433 \\
9 & 0.004\end{array}$ & $\begin{array}{cc}8 & 0.863 \\
& -0.433 \\
33 & 0.75 \\
4 & -0.008\end{array}$ & $\left.\begin{array}{ll}3 & 0 \\
3 & 0 \\
& 0 \\
8 & 0\end{array}\right)$ \\
\hline 0 & 0 & 0 & 0.01 & $\left(\begin{array}{c}1 \\
0 \\
0.006 \\
0.008\end{array}\right.$ & $\begin{array}{c}0 \\
1.002 \\
0 \\
0\end{array}$ & $\begin{array}{c}-0.032 \\
0 \\
0.996 \\
-0.12\end{array}$ & $\left.\begin{array}{c}-0.039 \\
0 \\
0.12 \\
0.996\end{array}\right)$ & $\left(\begin{array}{c}1 \\
-0.498 \\
0.866 \\
-0.086\end{array}\right.$ & $\begin{array}{c}-0.483 \\
0.248 \\
-0.432 \\
0.043\end{array}$ & $\begin{array}{c}0.837 \\
-0.43 \\
0.748 \\
-0.074\end{array}$ & $\left.\begin{array}{c}0.017 \\
-0.004 \\
0.015 \\
-0.001\end{array}\right)$ \\
\hline
\end{tabular}


Table 4: Experimental Mueller matrix given by the SMMP for vacuum and a linear partial polarizer at $30^{\circ}$ : theoretical, without corrections by $\phi_{w}, \phi_{2}, \phi_{3}$ and $\phi_{4}$ and with corrections by $\phi_{w}$, $\phi_{2}, \phi_{3}$ and $\phi_{4}$. All matrices are normalized by $m_{00}$. The experimental set-up is composed of two calcite plates $(\Delta n=0.166)$ for the coding system $(e=2.08 \mathrm{~mm} \pm 0.01 \mathrm{~mm})$ and two calcite plates for the decoding system $(e=10.4 \mathrm{~mm} \pm 0.01 \mathrm{~mm})$. The source is a broadband spectrum source with $\lambda_{0}=829 \mathrm{~nm}$, and the analysis window of the detection system is $\Delta \lambda=10 \mathrm{~nm}$ sampled with 512 pixels.

\begin{tabular}{|c|c|c|c|c|c|c|c|c|}
\hline & & Vacl & uum & & Linear & r partial & olarizer & at $30^{\circ}$ \\
\hline \multirow{4}{*}{ theoretical } & & $\left(\begin{array}{ll}1 & 0\end{array}\right.$ & $\begin{array}{ll}0 & 0\end{array}$ & & 1 & 0.50 & 0.866 & 0 \\
\hline & & $0 \quad 1$ & $\begin{array}{ll}0 & 0\end{array}$ & & 0.50 & 0.265 & 0.424 & 0 \\
\hline & & $\begin{array}{ll}0 & 0\end{array}$ & $\begin{array}{ll}1 & 0\end{array}$ & & 0.866 & $\begin{array}{ll}5 & 0.424\end{array}$ & 0.755 & 0 \\
\hline & & $\begin{array}{ll}0 & 0\end{array}$ & $\left.\begin{array}{ll}0 & 1\end{array}\right)$ & & 0 & 0 & 0 & 0.02 \\
\hline \multirow{4}{*}{ without corrections } & 1 & 0 & -0.067 & $-0.125)$ & 1 & -0.485 & -0.842 & -0.258 \\
\hline & -0.005 & 1 & 0.001 & -0.008 & 0.438 & -0.236 & -0.365 & -0.125 \\
\hline & -0.086 & 0.013 & 0.819 & -0.596 & -0.448 & 0.237 & 0.375 & 0.094 \\
\hline & 0.088 & 0.003 & 0.604 & 0.815 & -0.654 & 0.322 & 0.561 & 0.166 \\
\hline \multirow{4}{*}{ with corrections } & 1 & 0 & 0.003 & 0.003 & 1 & -0.480 & -0.863 & -0.005 \\
\hline & 0.003 & 1 & 0.003 & 0.007 & -0.490 & 0.261 & 0.423 & 0.016 \\
\hline & -0.002 & 0.010 & 1.004 & 0.017 & -0.868 & 0.434 & 0.756 & -0.013 \\
\hline & 0 & -0.005 & -0.018 & 0.995 & -0.003 & -0.015 & 0.013 & 0.015 \\
\hline
\end{tabular}


Table 5: Simulation of the influence of the misalignment errors, $\Delta \theta_{1}, \Delta \theta_{2}, \Delta \theta_{3}, \Delta \theta_{4}$ and $\Delta \theta_{p o l}$, on the Mueller matrix for vacuum.

\begin{tabular}{|c|c|c|c|c|c|c|c|c|}
\hline$\Delta \theta_{\mathrm{pol}}\left({ }^{\circ}\right)$ & $\Delta \theta_{1}\left({ }^{\circ}\right)$ & $\Delta \theta_{2}\left(^{\circ}\right)$ & $\Delta \theta_{3}\left(^{\circ}\right)$ & $\Delta \theta_{4}\left(^{\circ}\right)$ & \multicolumn{4}{|c|}{ Mueller Matrix for vacuum } \\
\hline 0 & 0 & 0 & 0 & 0 & & $\left(\begin{array}{ll}1 & 0 \\
0 & 1 \\
0 & 0 \\
0 & 0\end{array}\right.$ & $\left.\begin{array}{ll}0 & 0 \\
0 & 0 \\
1 & 0 \\
0 & 1\end{array}\right)$ & \\
\hline 0.5 & 0 & 0 & 0 & 0 & $\left(\begin{array}{l}1 \\
0 \\
0 \\
0\end{array}\right.$ & $\begin{array}{cc}0 & 0 \\
1 & 0.01 \\
0 & 1 \\
0 & 0\end{array}$ & $\begin{array}{cc} & 0 \\
15 & -0.0 \\
& 0 \\
& 1\end{array}$ & 008 \\
\hline 0 & 0.5 & 0 & 0 & 0 & $\left(\begin{array}{c}1 \\
0 \\
0.015 \\
-0.008\end{array}\right.$ & $\begin{array}{c}0 \\
1 \\
-0.015 \\
0.008\end{array}$ & $\begin{array}{c}0 \\
0 \\
0.982 \\
0\end{array}$ & $\left.\begin{array}{c}0 \\
0 \\
0 \\
0.982\end{array}\right)$ \\
\hline 0 & 0 & 0.5 & 0 & 0 & $\left(\begin{array}{c}1 \\
0 \\
-0.015 \\
0.008\end{array}\right.$ & $\begin{array}{c}0 \\
1.003 \\
0.017 \\
0\end{array}$ & $\begin{array}{c}0 \\
-0.018 \\
1.017 \\
0\end{array}$ & $\begin{array}{c}0 \\
0 \\
0 \\
1.017\end{array}$ \\
\hline 0 & 0 & 0 & 0.5 & 0 & $\left(\begin{array}{lr}1 & \\
0 & \\
0 & -0 \\
0 & \end{array}\right.$ & $\begin{array}{cc}0 & 0 . \\
1 & 0 . \\
0.018 & 1 . \\
0 & \end{array}$ & $\begin{array}{cc}.015 & - \\
.017 & \\
.017 & \\
0 & 1\end{array}$ & $\left.\begin{array}{c}-0.008 \\
0 \\
0 \\
1.017\end{array}\right)$ \\
\hline 0 & 0 & 0 & 0 & 0.5 & $\left(\begin{array}{l}1 \\
0 \\
0 \\
0\end{array}\right.$ & $\begin{array}{cc}0 & -0.0 \\
1 & -0.0 \\
0 & 0.98 \\
0 & 0\end{array}$ & $\begin{array}{lr}015 & 0.0 \\
015 & 0.0 \\
82 & 0 \\
& 0.9\end{array}$ & $\left.\begin{array}{l}008 \\
008 \\
0 \\
982\end{array}\right)$ \\
\hline 0.5 & 0.5 & -0.5 & 0.5 & 0.5 & $\left(\begin{array}{c}1 \\
0.002 \\
0.031 \\
-0.017\end{array}\right.$ & $\begin{array}{c}0 \\
0.998 \\
-0.050 \\
0.008\end{array}$ & $\begin{array}{c}0 \\
0.034 \\
0.964 \\
0\end{array}$ & $\begin{array}{cc} & 0 \\
4 & 0 \\
4 & 0 \\
& 0.965\end{array}$ \\
\hline 0.1 & 0.1 & -0.1 & 0.1 & 0.1 & $\left(\begin{array}{c}1 \\
0 \\
0.006 \\
-0.003\end{array}\right.$ & $\begin{array}{c}0 \\
1 \\
-0.01 \\
0.002 \\
\end{array}$ & $\begin{array}{c}0 \\
0.007 \\
0.993 \\
0 \\
\end{array}$ & $\begin{array}{c}0 \\
0 \\
0 \\
0.993\end{array}$ \\
\hline
\end{tabular}


Table 6: Simulation of a quarter wave plate $\left(R=90^{\circ}, \alpha=20^{\circ}\right)$ at different orders. Depolarization index $P_{D}$, retardance $R$ and azimuthal angle $\alpha$ are calculated. The ratio between the evolution with the wavelength of the quarter wave plate retardance and the evolution with the wavelength of the reference coding plate retardance is given.

\begin{tabular}{cccc}
\hline $\begin{array}{c}\Delta \phi_{\lambda / 4} / \Delta \phi_{\text {coding }} \\
(\%)\end{array}$ & $\mathrm{P}_{\mathrm{D}}$ & $\mathrm{R}\left(^{\circ}\right)$ & $\alpha\left(^{\circ}\right)$ \\
\hline 0.055 & 0.999 & 89.99 & 19.99 \\
0.275 & 0.999 & 89.94 & 19.99 \\
0.55 & 0.998 & 89.84 & 19.97 \\
1.1 & 0.995 & 89.66 & 19.93 \\
2.75 & 0.975 & 89.55 & 19.58 \\
5.5 & 0.917 & 82.88 & 18.64 \\
\hline
\end{tabular}


Table 7: Experimental Mueller matrix for the quartz wave plate: theoretical, with no correction, and with correction.

\begin{tabular}{cccc}
\hline \multicolumn{3}{c}{ theoretical } & without correction \\
\hline$\left(\begin{array}{cccc}1 & 0 & 0 & 0 \\
0 & 0.914 & -0.407 & 0 \\
0 & 0.407 & 0.914 & 0 \\
0 & 0 & 0 & 1\end{array}\right) \quad\left(\begin{array}{cccccccc}1 & -0.056 & 0.018 & -0.006 \\
-0.019 & 0.926 & -0.386 & -0.012 \\
0.021 & 0.464 & 0.911 & 0.03 \\
-0.019 & -0.147 & 0.032 & 1.013\end{array}\right) \quad\left(\begin{array}{ccccc}1 & 0.023 & 0.022 & -0.009 \\
-0.005 & 0.919 & -0.399 & -0.006 \\
0.010 & 0.379 & 0.907 & 0.016 \\
-0.01 & -0.014 & 0.017 & 1.004\end{array}\right)$ \\
\\
\end{tabular}

